\title{
Pembrolizumab-Induced Lichenoid Dermatitis in a Patient With Metastatic Cancer of Unknown Primary
}

\author{
Ashish Sethi ${ }^{1}$, Moses Raj ${ }^{2}$ \\ 1. Internal Medicine, Allegheny Health Network, Pittsburgh, USA 2. Hematology \& Oncology, Allegheny Health \\ Network, Pittsburgh, USA
}

Corresponding author: Ashish Sethi, sethi_ash97@rediffmail.com

\begin{abstract}
Pembrolizumab is an immune checkpoint inhibitor approved for use in many cancer types such as non-small cell lung cancer (NSCLC), metastatic melanoma, head and neck cancers, hepatocellular carcinoma, and renal cell carcinoma. There are many reported cases of patients on immunotherapy who have discontinued treatment due to the development of immune-related adverse effects (irAE). Recognition of the histopathologic patterns of dermatologic toxicities due to immunotherapy will become increasingly important for ensuring appropriate management and optimal patient care. Here, we present a case of a 72year-old man with metastatic carcinoma of unknown primary origin treated with pembrolizumab who developed an immune-related cutaneous adverse event (ircAE) in the form of lichenoid dermatitis.
\end{abstract}

Review began 02/28/2021 Review ended 03/07/2021 Published 03/08/2021

\section{○ Copyright 2021}

Sethi et al. This is an open access article distributed under the terms of the Creative Commons Attribution License CC-BY 4.0., which permits unrestricted use, distribution, and reproduction in any medium, provided the original author and source are credited.
Categories: Dermatology, Internal Medicine, Oncology

Keywords: pembrolizumab, lichenoid drug eruption, supraclavicular lymph node, metastatic cancer of unknown primary, cdx2, ck7, lichen planus, immunotherapy-related adverse events, lichenoid dermatitis, psoriasiform rash

\section{Introduction}

Pembrolizumab is a humanized monoclonal antibody that inhibits the interaction between the programmed death-ligand 1(PD-L1) receptor on T-cells and the PD-L1 and PD-L2 ligands on tumor cells [1]. The interaction of this immunotherapy subtype promotes T-cell reactivation and restores the immune response causing T-cells to attack healthy cells, leading to various autoimmune diseases referred to as immunerelated adverse effects (irAE).

Cutaneous toxicities inflict many challenges. A rash is the most common cutaneous toxicity associated with pembrolizumab. Also, it can cause a variety of inflammatory conditions like spongiotic, psoriasiform, and lichenoid dermatitides, mimicking eczema, psoriasis, and lichen planus, respectively. The clinical presentations may be focal or diffuse, including flexural and erythrodermic variants. Pruritus associated with various dermatitides can be severe.

Vitiligo, as an immune-related cutaneous adverse event (ircAE) presents in the form of well-demarcated depigmented macules or patches. Besides contrasting presentation, time to onset varies greatly among such rashes, as vitiligo can appear late after treatment initiation. However, inflammatory dermatoses usually occur within the first one to two cycles into the immunotherapy or pembrolizumab treatment. This requires constant vigilance for signs and symptoms of different cutaneous toxicities. As a result, ircAE has been recognized as a contributing factor to treatment noncompliance, discontinuation, or dose modification. However, with targeted systemic therapies now being available for eczema and psoriasis, correlating the inflammatory patterns of the cutaneous eruptions with the inflammatory patterns that they mimic can help may in more efficacious treatments and fewer drug interruptions and dose modifications. More importantly, it can increase the compliance and efficacy of the pembrolizumab and other immunotherapies.

We report an unusual case of metastasis of unknown primary origin treated with pembrolizumab who developed a widespread lichenoid dermatitis or lichenoid drug eruption.

\section{Case Presentation}

A 72-year-old male presented with a pruritic rash on the trunk and extremities of one-week duration. His medical history included metastasis of unknown primary, hypertension, type 2 diabetes mellitus, and hypercholesterolemia.

His initial presentation of chronic arthritic pain and lumps in his neck was evaluated by radiological imaging and biopsy. Computed tomography (CT) of the chest and neck revealed a right upper lobe lung mass with mediastinal and supraclavicular lymphadenopathy (Figures 1-2). Subsequently, a fluorodeoxyglucose (FDG)-positron emission tomography (PET) scan illustrated widespread metastatic disease with higher FDG 


\section{Cureus}

uptake at the right sacrum, lung mass, and left supraclavicular lymph node (SCLN) (Figures 3-5).

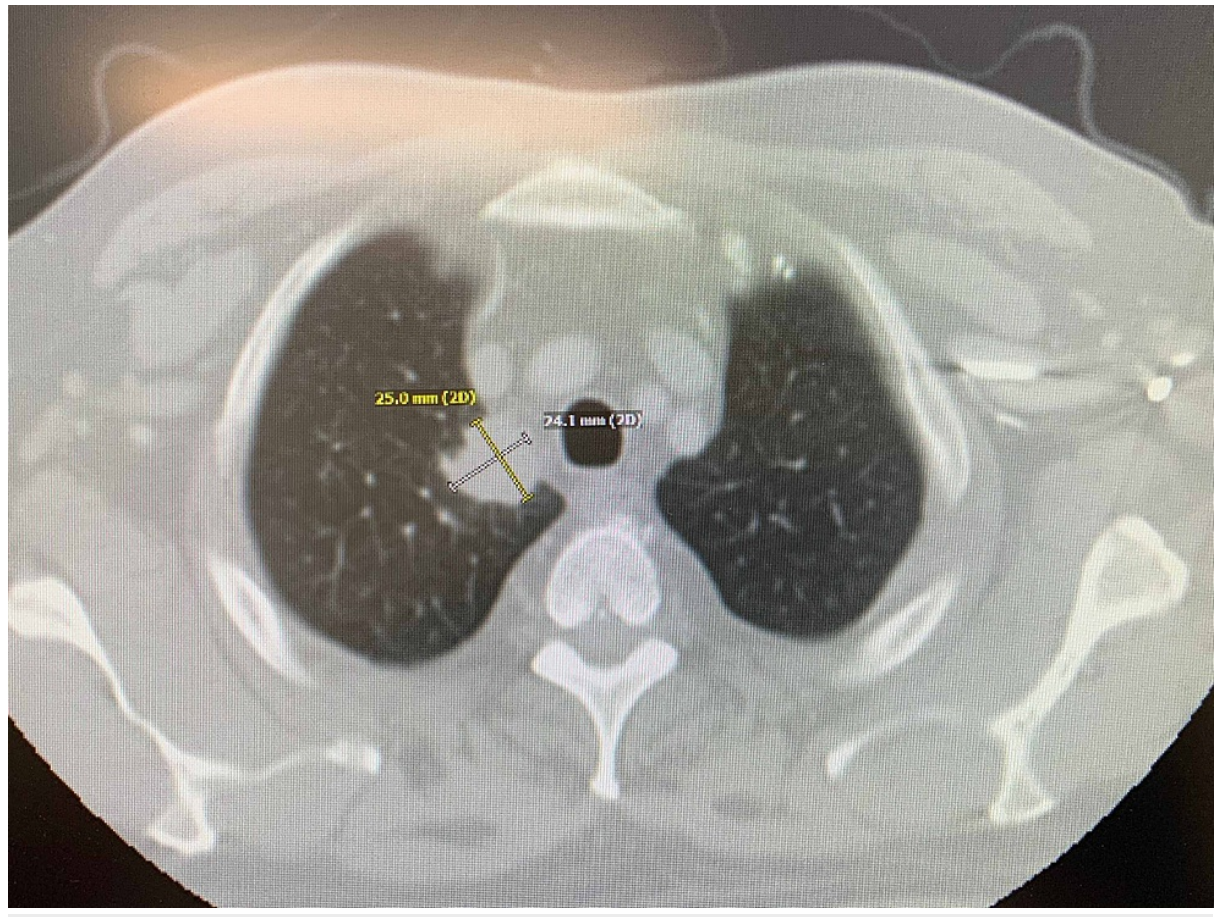

\section{FIGURE 1: CT chest with contrast}

Irregular mass concerning for malignancy abutting the right mediastinum within this upper lobe measuring $2.3 \times 3 \mathrm{~cm}$.

CT: computed tomography

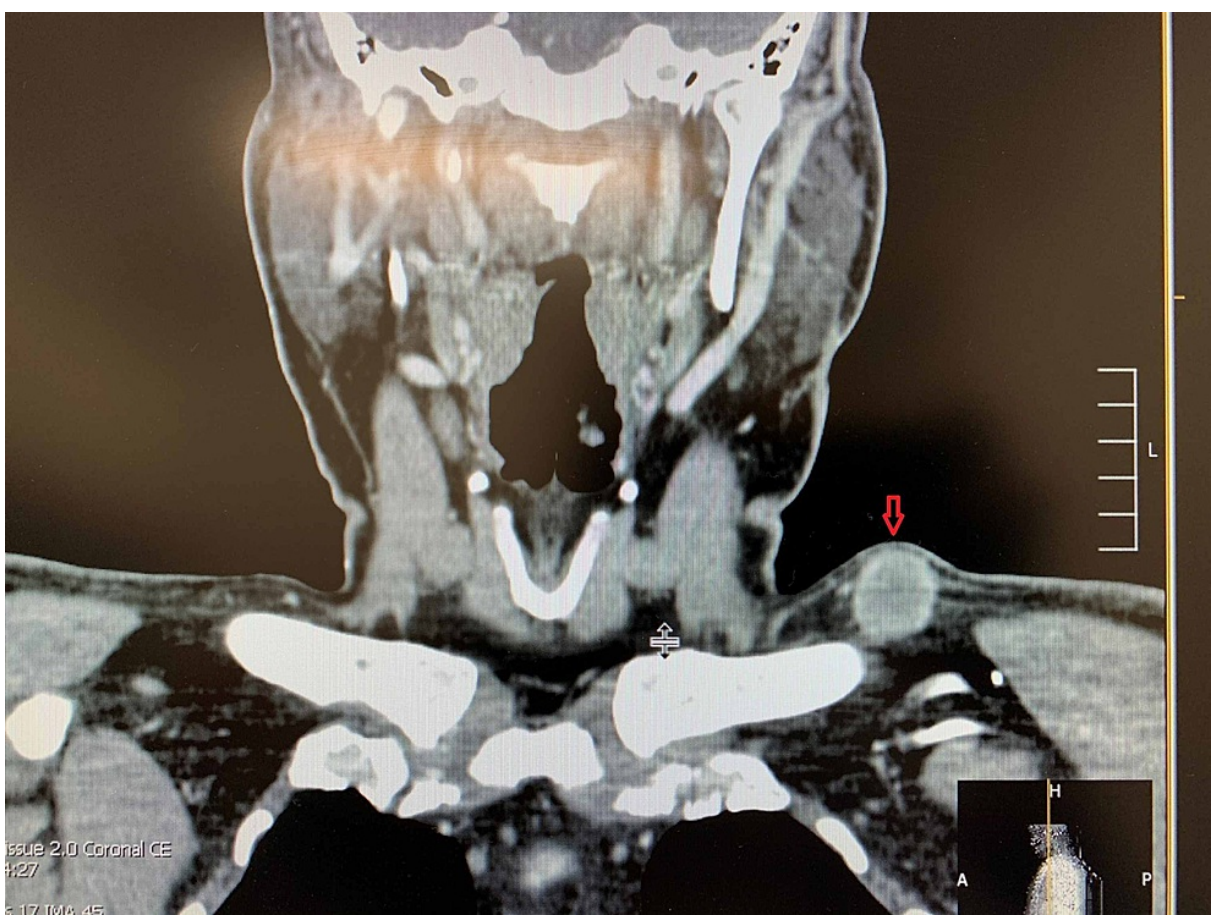

FIGURE 2: CT neck

Red downward arrow: enlarged left supraclavicular lymph node

CT: computed tomography 


\section{Cureus}

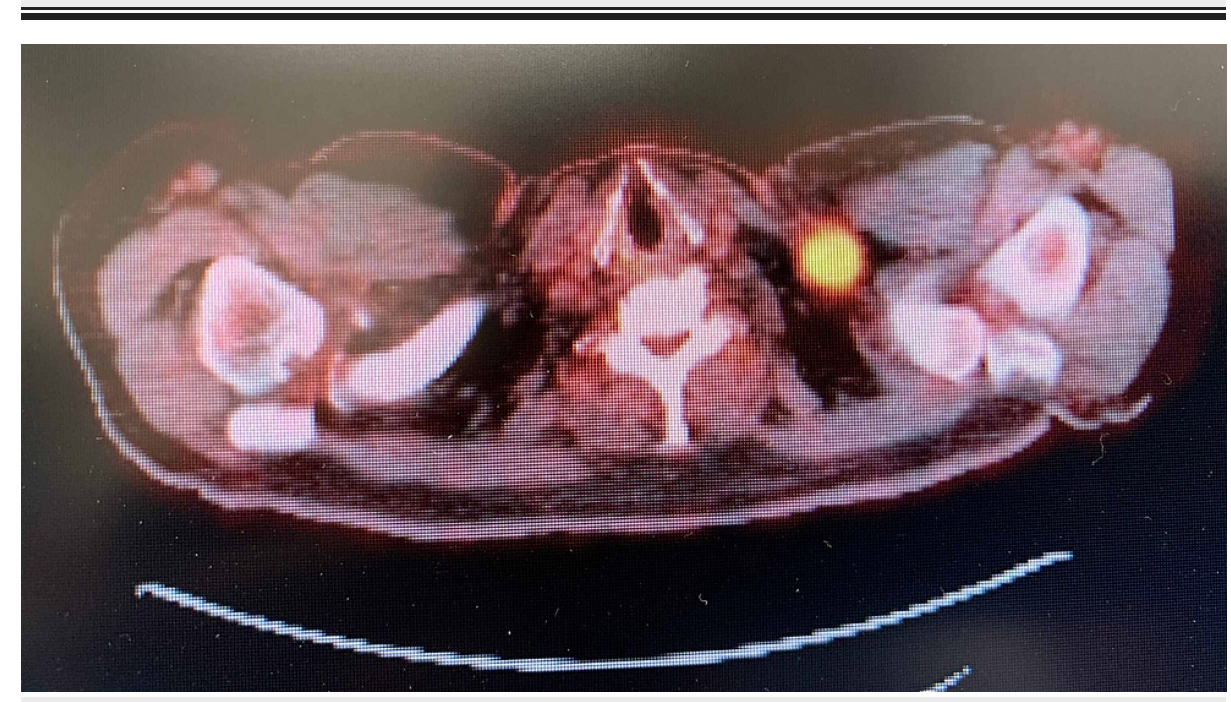

\section{FIGURE 3: PET scan}

Enlarged, hypermetabolic lateral left supraclavicular lymph node with maximum SUV of 6.8

SUV: standardized uptake value; PET: positron emission tomography

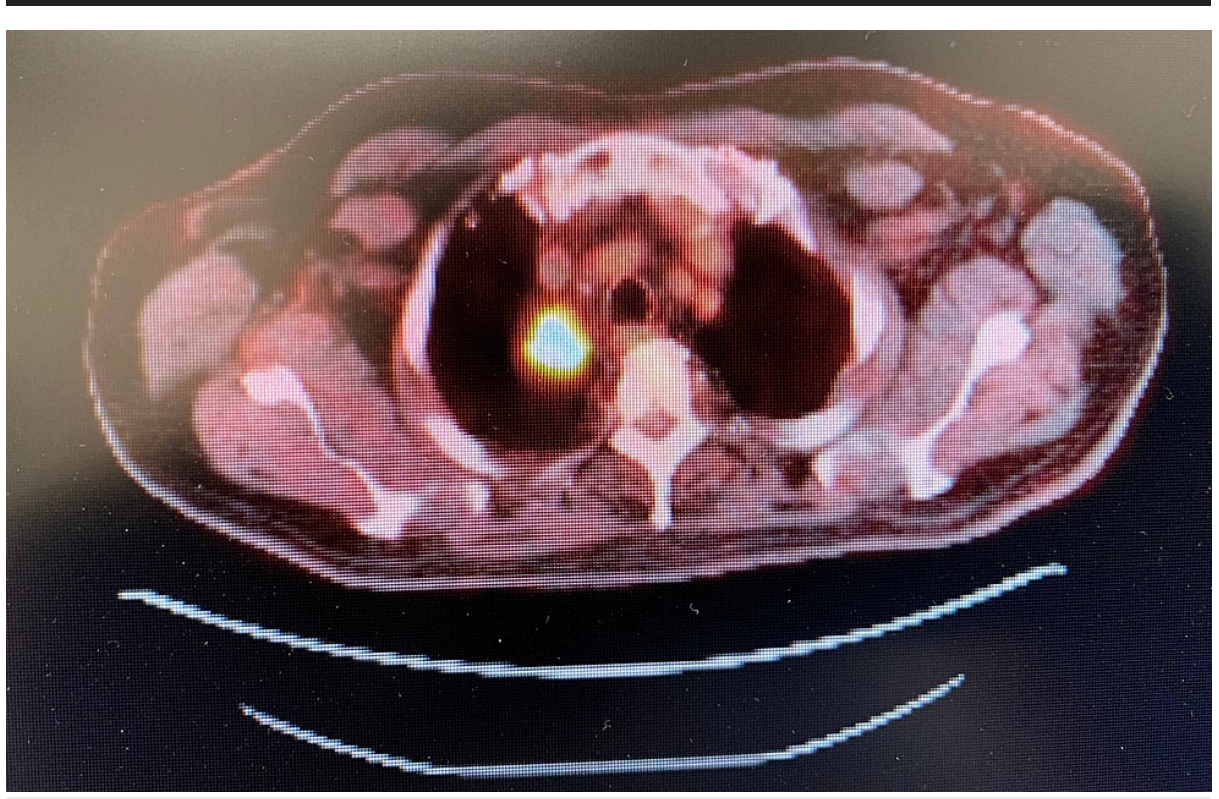

\section{FIGURE 4: PET scan}

Hypermetabolic right lung mass abutting the mediastinum, with SUV measuring 14.4

SUV: standardized uptake value; PET: positron emission tomography 


\section{Cureus}

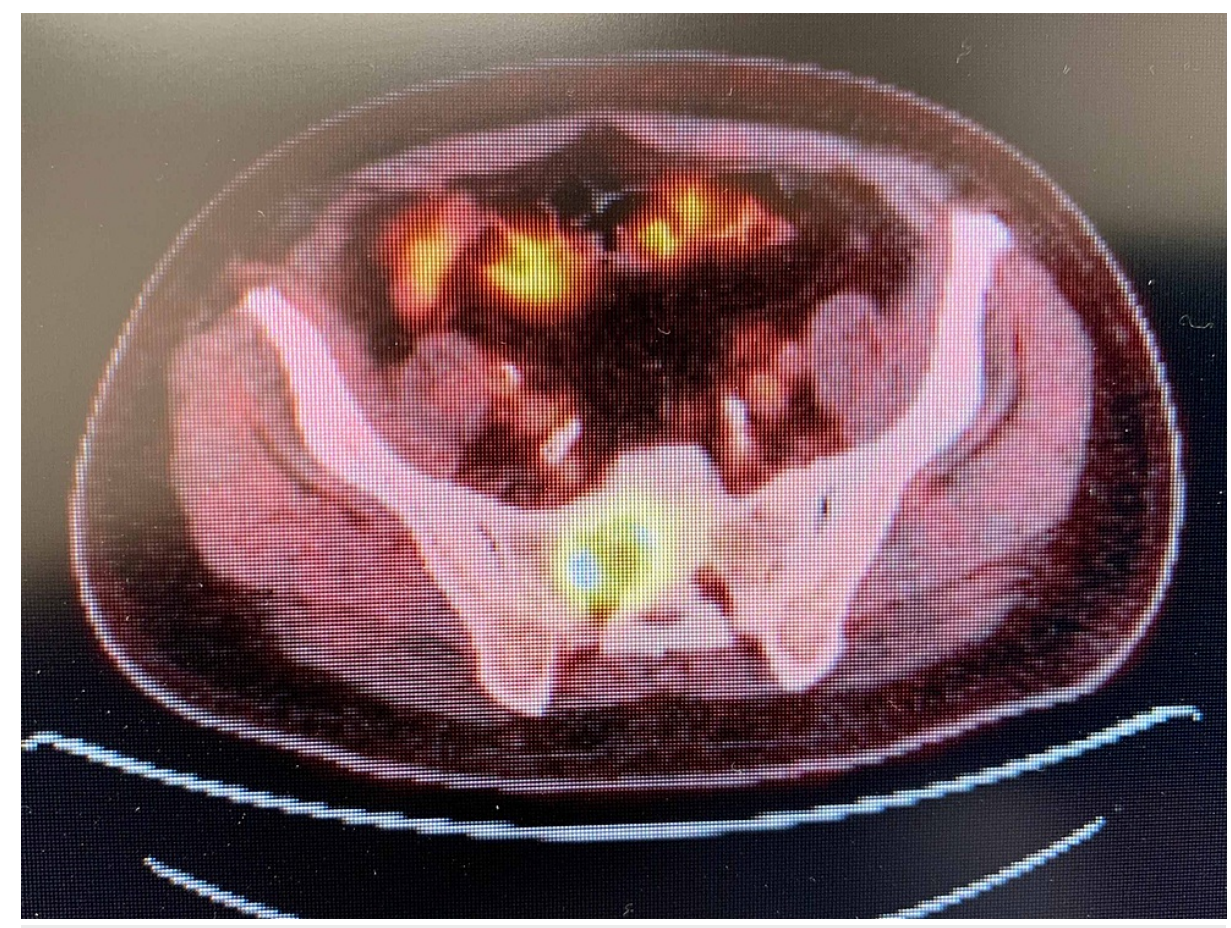

FIGURE 5: PET scan

A large lytic lesion is identified at the right sacrum, with a maximum SUV of 11.4

SUV: standardized uptake value; PET: positron emission tomography

The patient underwent an excisional biopsy of the enlarged left supraclavicular lymph node. Biopsy revealed metastatic, poorly differentiated carcinoma with necrosis and extra-nodal extension.

Immunohistochemistry (IHC) performed on the specimen was positive for cytokeratin 7 and CDX2 (Figure 6) and negative for cytokeratin 20, p40, p63, synaptophysin, chromogranin, TTF-1, GATA-3, and PAX8,

favoring adenocarcinoma from probably the upper gastrointestinal tract (GIT) as the primary source of origin (Table 1). His liver function tests (LFTs) remained within normal range throughout the clinical course and the carcinoembryonic antigen (CEA) levels were 13 nanograms per milliliter $(\mathrm{ng} / \mathrm{ml}$ ) (Normal CEA: 0-3 $\mathrm{ng} / \mathrm{ml})$. He was initially treated with modified FOLFIRINOX based on his clinical presentation and diagnostic evaluation.

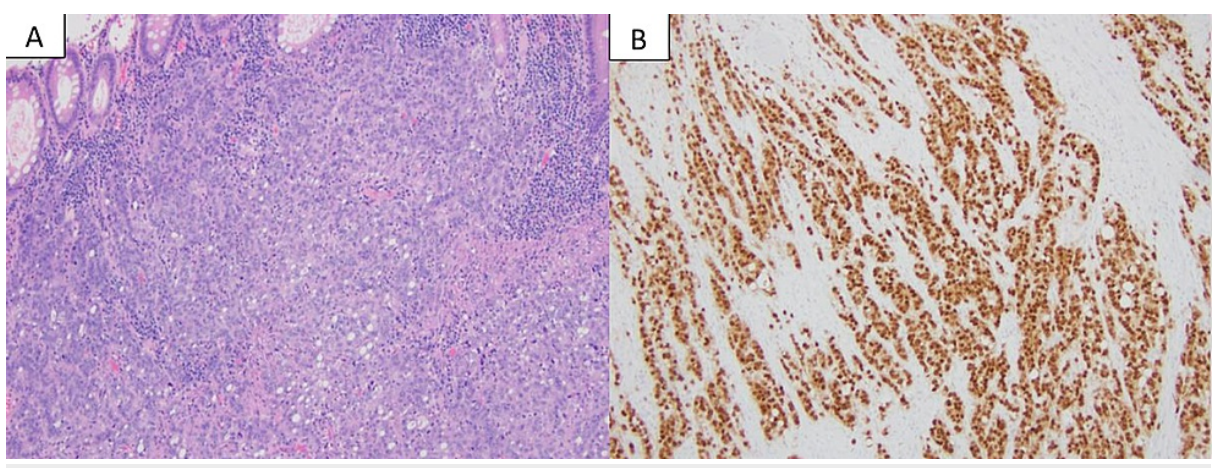

\section{FIGURE 6: Pathological analysis of the surgical specimen}

Resection reveals a poorly differentiated adenocarcinoma with a solid and vague glandular growth pattern with many tumor-infiltrating lymphocytes, features of mismatch repair-deficient carcinoma (A, H\&E stain). The tumor cells are strongly positive for CDX2 (B, immunostain), supporting a diagnosis of colonic adenocarcinoma.

H\&E: hematoxylin and eosin

Permission was taken from the original publisher; adapted from De Leo et al. [2]. 


\section{Cureus}

Immunohistochemical Stains

CK 7 positive and CK 20 negative

CDX2 positive

$\mathrm{p} 40$ and $\mathrm{p} 63$ negative

Synaptophysin and Chromogranin negative

TTF- 1 negative

GATA 3 negative

PAX 9 negative
Inferences

Primary is located "above" the diaphragm or from a gynecological origin

A gastrointestinal tract, biliary, or pancreatic origin

Not a squamous cell carcinoma

Not a neuroendocrine tumor

Not a lung adenocarcinoma/small cell primary or a thyroid tumor

Not urothelial or breast primary

Not kidney, thymic, thyroid, and Mullerian as primary

\section{TABLE 1: Immunohistochemical stains and analysis}

CK: cytokeratin; CDX2: caudal type homeobox 2; TTF-1: thyroid transcription factor 1; PAX9: paired box gene 9

His PDL 1 status was $60 \%$ on lymph node biopsy, which was a reasonable choice for starting immunotherapy with pembrolizumab. Molecular studies performed for epidermal growth factor receptor (EGFR), echinoderm microtubule-associated protein-like 4 (EML4), anaplastic lymphoma kinase (ALK) genes, and next-

generation sequencing (NGS) were negative. Later, the treatment plan was modified and pembrolizumab was added to the concurrent chemo-radiation regimen with carboplatin and paclitaxel. He also received subsequent radiation therapy to the thoracic spine, left pleural metastasis, right tibia, and thenar eminence of the left hand in view of his disease spectrum and pain at these sites.

After the second cycle of pembrolizumab with a cumulative dose of $400 \mathrm{mg}$, the patient reported multiple, similar-appearing erythematous, firm papules and plaques, which were pruritic and progressive in nature in the chest, gluteal region, and both upper and lower extremities (Figures 7-11). It was initially diagnosed as psoriasiform drug eruption and started on oral prednisolone $20 \mathrm{mg}$ once a day and a urea cream $40 \%$ twice a day, and his pembrolizumab was held. After no major improvement in the rash from the treatment, punch biopsy performed from right forearm lesions confirmed the diagnosis of drug-induced lichenoid dermatitis. The histological features on skin biopsy illustrated basal vacuolization of the dermal-epidermal junction and perifollicular lichenoid inflammatory infiltrations. 


\section{Cureus}

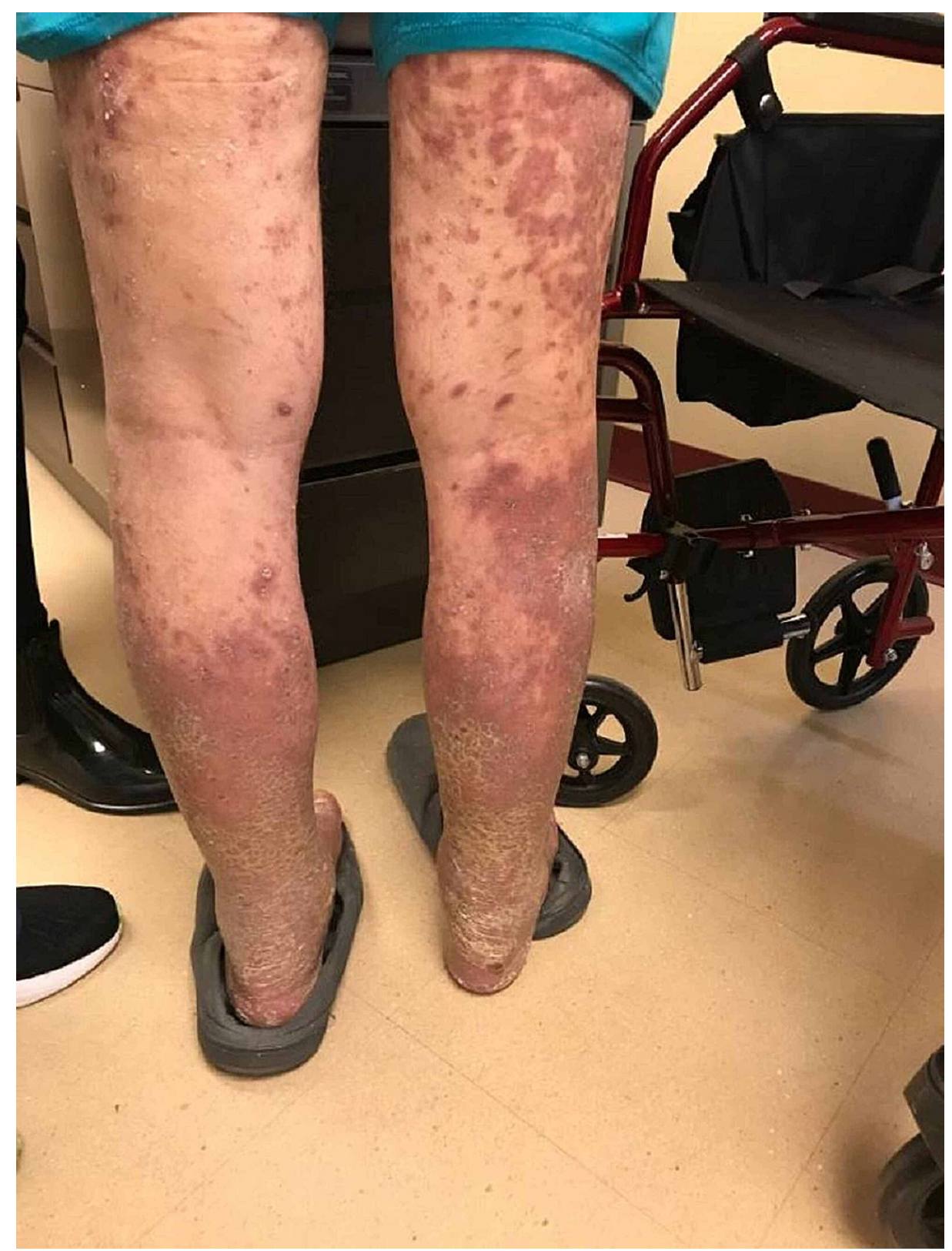

FIGURE 7: Cutaneous manifestations of lichenoid eruption - lower extremities

-Pembrolizumab-induced

-Erythematous to violaceous eruption of hyperkeratotic papules and plaques on the legs 


\section{Cureus}

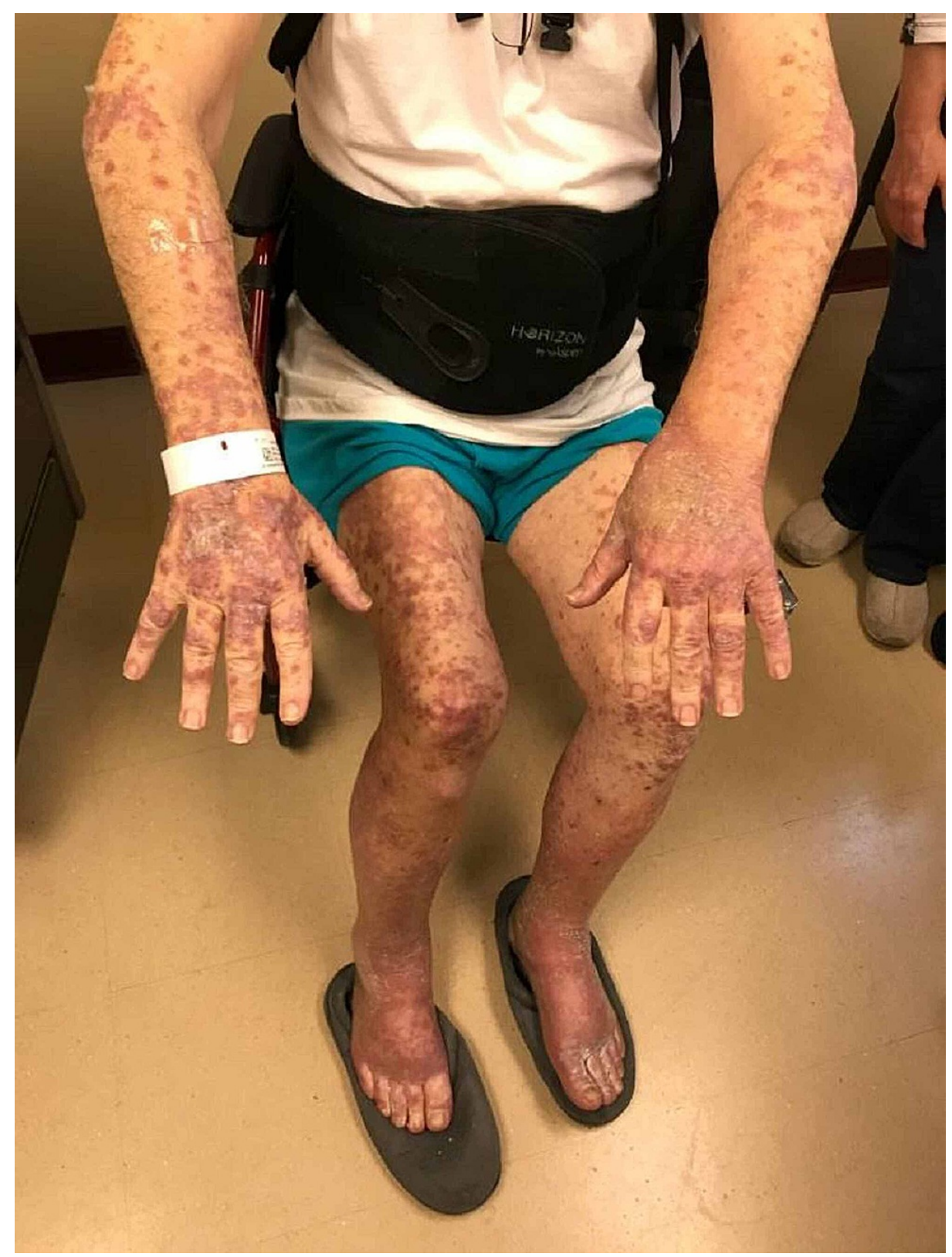

FIGURE 8: Lichenoid eruption - upper and lower extremities

Pembrolizumab-induced 


\section{Cureus}

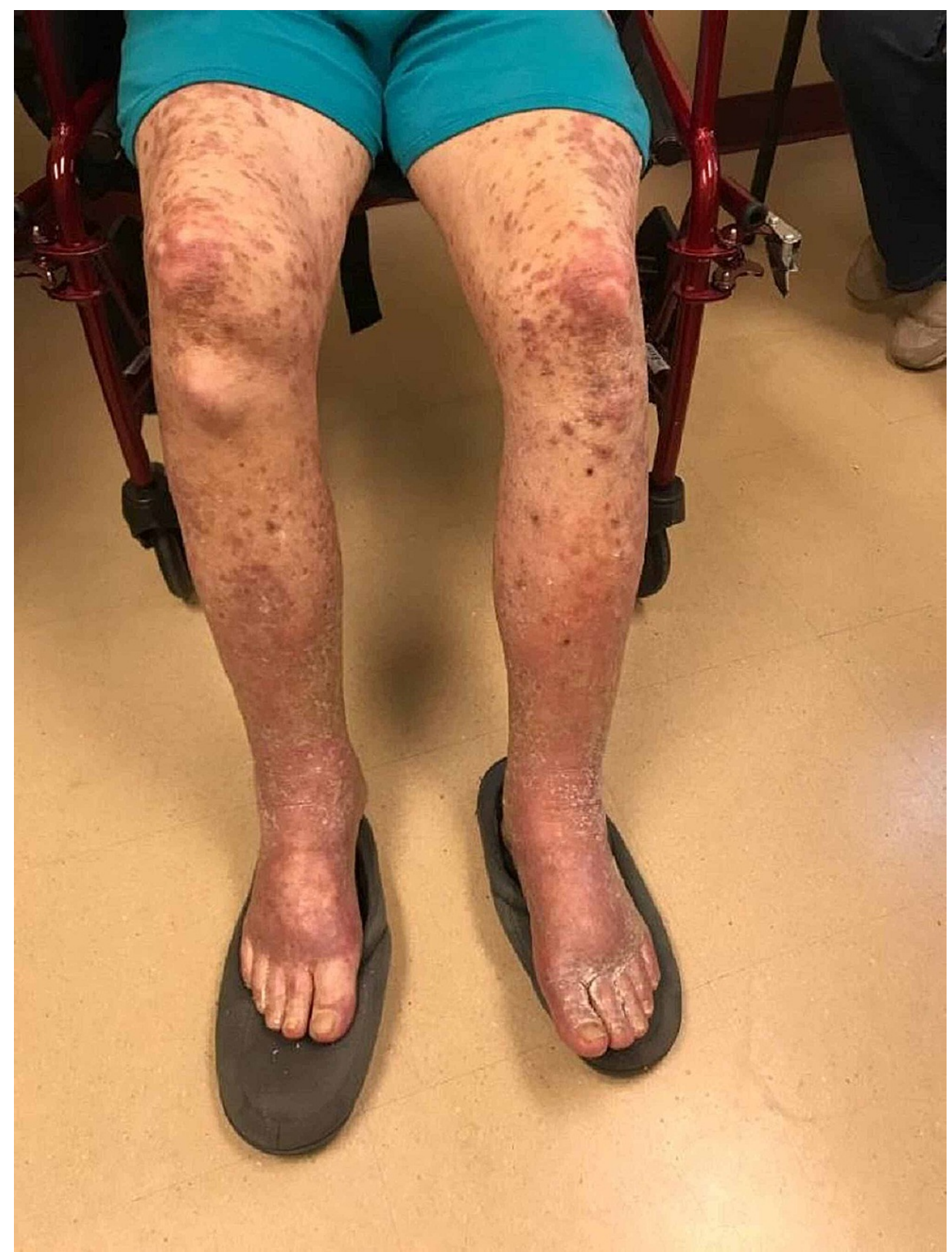

FIGURE 9: Lichenoid eruption - lower extremities

Pembrolizumab-induced 


\section{Cureus}

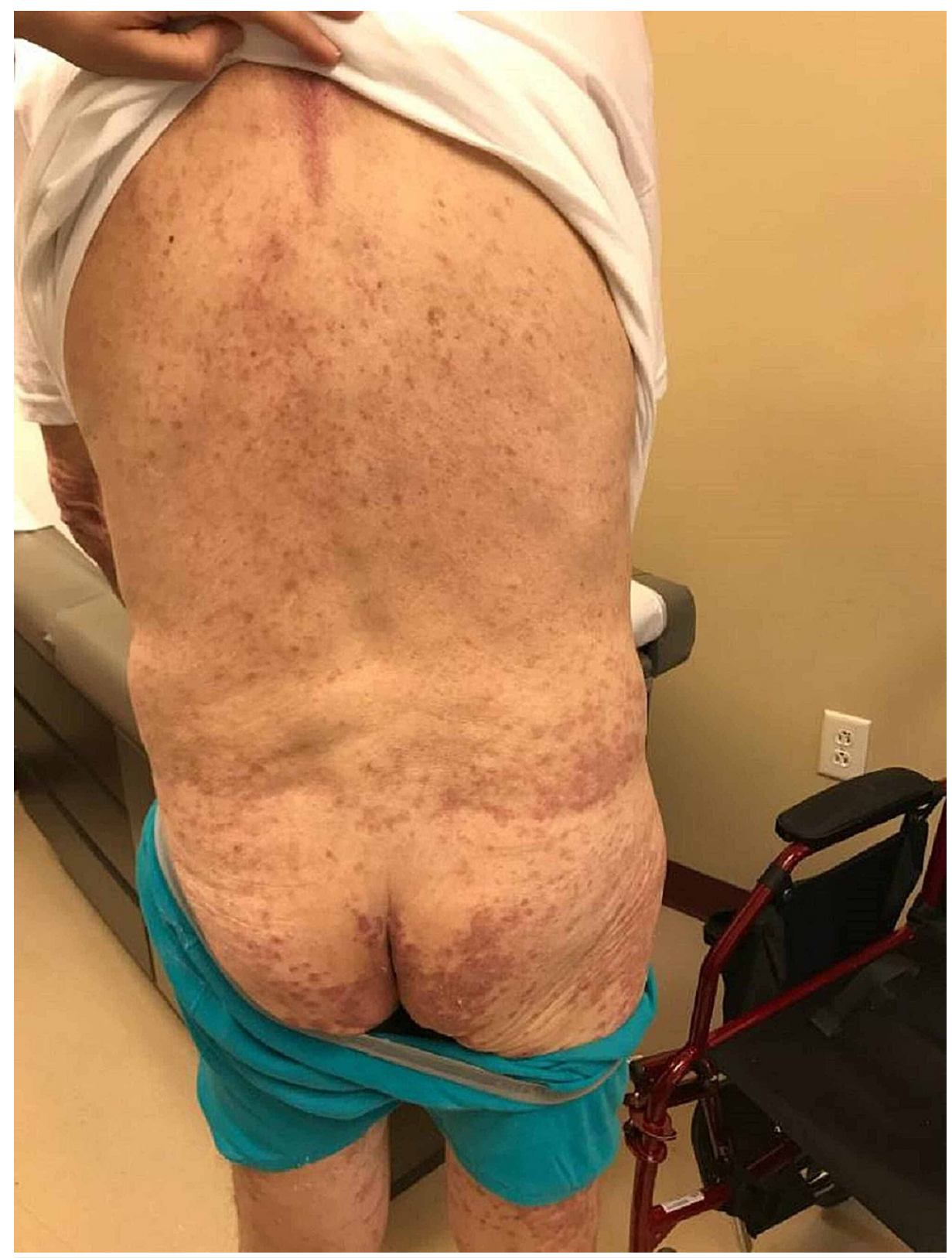

FIGURE 10: Lichenoid eruption - gluteal region and back

Pembrolizumab-induced 


\section{Cureus}

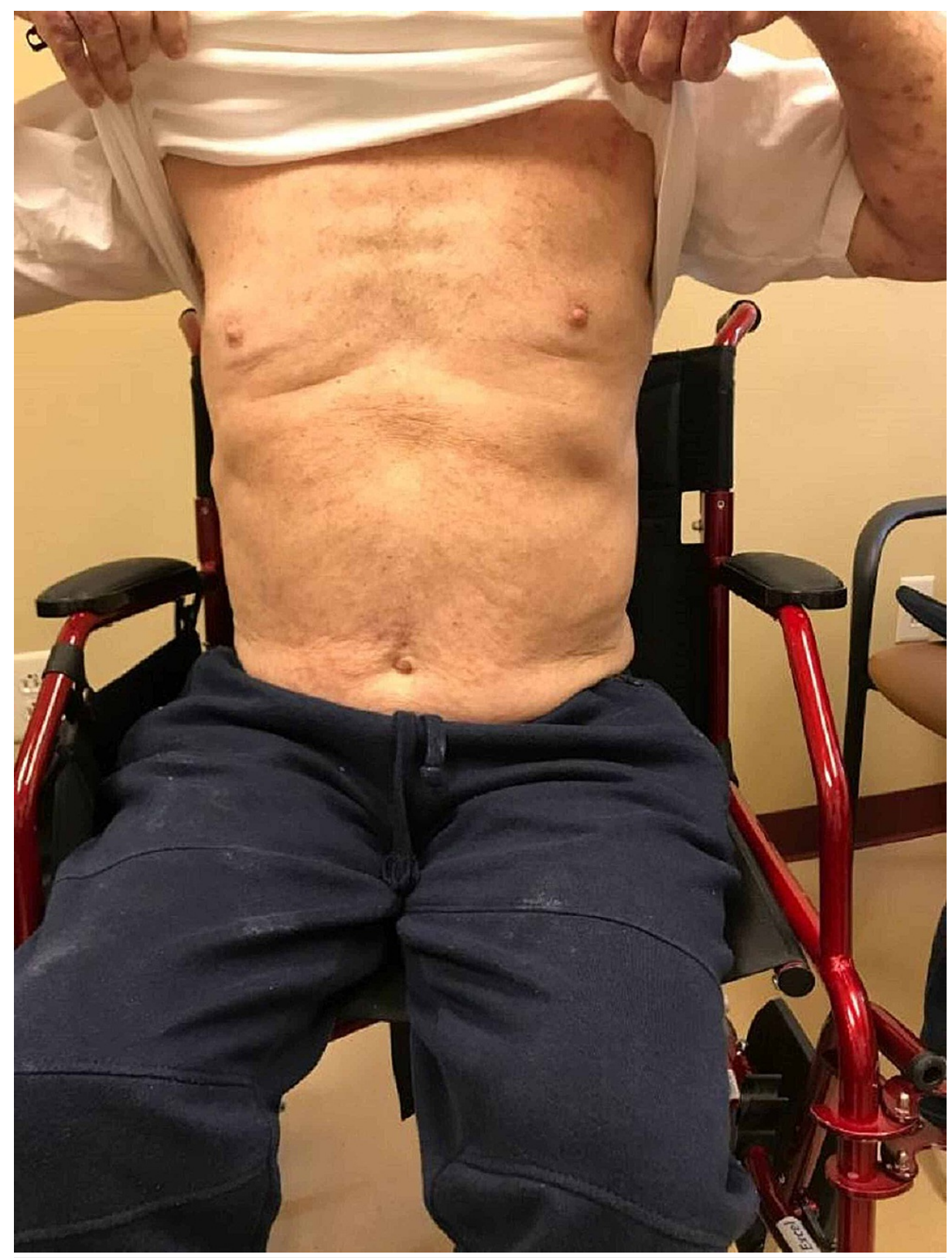

\section{FIGURE 11: Lichenoid eruption - chest and abdomen}

Pembrolizumab-induced

Faintly erythematous, papules and plaques

\section{Discussion}

The duration and onset of lichenoid drug reactions are often dependent on the causative agent and dosage. The pathogenesis of lichenoid drug reaction is not well-understood. T-cells, keratinocytes, dendritic cells, and endothelial cells, which express activation markers, costimulatory molecules, and adhesion molecules such as microhistocompatibility (MHC) class-II molecules, L-selectin, intercellular adhesion molecule (ICAM) 1 are thought to be involved in the inflammatory reaction that ultimately leads to the apoptosis of basal keratinocyte [3].

Lichenoid drug eruptions are mostly seen in individuals between the age of 57 to 66 years and can have a latent period of one year or more [4]. The clinical presentation (Table 2) and pathological features of lichenoid drug eruptions are similar to lichen planus. Both conditions exhibit erythematous papules and plaques; however, lichenoid drug eruptions may be scaly, highly pruritic, and resolve with greater residual hyperpigmentation [4-5]. Also, Wickham's striae - a lacy, white network of streaks and the involvement of other mucosal areas are observed less frequently in drug-induced lesions [4-5]. Compared to the flexor surface involvement on extremities with idiopathic lichen planus, lichenoid drug eruption present in a 


\section{Cureus}

photo-distributed or symmetric pattern [4].

\section{Lichenoid drug eruption}

Extensive rash: symmetrical over trunks and extremities

Photodistribution: rash in areas exposed to the sun

Rash may be scaly mimicking eczema or psoriasis

Wickam striae usually not seen

Nail and mucus membrane involvement is rare

Pigmentation seen after active rash has cleared

\section{TABLE 2: Lichenoid drug eruption features}

In addition, acanthosis, hypergranulosis, and hyperkeratosis are some of the common characteristic features seen in both lichen planus and lichenoid drug eruptions [6]. However, infiltration of eosinophils in the dermis can delineate lichenoid drug eruption from lichen planus [6].

Lichenoid reactions are chronic inflammatory, T-cell-mediated reactions to an antigen. There are many types of drugs that may trigger this condition (Table 3). In contrast, lichen planus can be associated with systemic conditions such as diabetes mellitus and hepatitis infections.

\section{Anticonvulsants such as carbamazepine or phenytoin}

Anti-hypertensives like ACE inhibitors, beta-blockers, nifedipine

Chemotherapy drugs such as fluorouracil, hydroxyurea, or imatinib

Diuretics, like furosemide, hydrochlorthiazide, and spironolactone

HMG-CoA reductase inhibitors

NSAIDs

Hypoglycemic agents

Proton pump inhibitors

Sildenafil citrate

Sulfa drugs, including dapsone, sulfasalazine

\section{Tetracycline}

Antitubercular drugs

Tumor necrosis factor antagonists : adalimumab, infliximab

Interferon alfa

Hydroxychloroquine

Misoprostol

Ketoconazole

Phenothiazine derivatives

\section{TABLE 3: Drugs - lichenoid eruption or reaction}

ACE: angiotensin-converting enzyme; NSAIDs: non-steroidal anti-inflammatory drugs 
Lichenoid drug eruptions are usually less likely to resolve and may require discontinuation of the causative agent in addition to topical or oral corticosteroid therapy based on severity. When lichenoid drug eruptions are suspected, the most important step is to discontinue the medication; however, resolution is slow and may take several months to up to 1 year.

Pembrolizumab, a type of immunotherapy, targets PD-1 and has been approved for managing various malignancies viz. melanoma, non-small-cell lung cancer (NSCLC), head and neck squamous cancers, urothelial carcinoma, gastric adenocarcinoma, mismatch-repair-deficient solid tumors, and Hodgkin's lymphoma. Cutaneous toxicities are seen in $30 \%$ to $50 \%$ of patients treated with various immunotherapies like nivolumab, ipilimumab, and pembrolizumab, etc. [7]. It can manifest as pruritus, vitiligo, lichenoid dermatitis, psoriasiform eruptions, and bullous pemphigoid. Our understanding of cutaneous toxicities stems mostly from ipilimumab use where the overall incidence ranges between the $37 \%$ and $70 \%$ range for all grades and $1 \%$ to $3 \%$ for grade 3 or more [8-9]. However, the incidence of grade 3 or higher toxicities of antiPD-1 agents is the same as with ipilimumab. Although cutaneous toxicities are transient, they can cause significant morbidity and non-compliance with the ongoing treatment regimen, thus leading to impairment of the patient's health-related quality of life. A similar case of intense lichenoid inflammation and hypergranulosis has been reported in a patient on avelumab, which is also an anti-PD-L1 therapy [10].

The possibility of acute radiation dermatitis at radiation sites causing erythema, hyperpigmentation, pruritus, and burning sensation of skin was also considered in our patient. However, the more conventional, sustained hyperpigmentation or erythema associated with radiotherapy typically does not occur until two to four weeks into treatment [11].

Our case shows that pembrolizumab can induce lichenoid eruption in form of lichenoid dermatitis. It is often characterized by large monomorphic lesions mimicking psoriasiform appearance, with desquamation and crusting that corresponds with the findings observed in our case.

\section{Conclusions}

At present, knowledge of the incidence and diagnosis of various cutaneous irAEs is limited. Discontinuation of immunotherapy remains the only option. As the optimal duration of immunotherapy treatment has not been established in cancer, it is uncertain whether the efficacy of these drugs persists after their discontinuation. Collaboration between oncologists and dermatologists is important in order to understand the spectrum of cutaneous irAEs, optimize their treatment, and define the appropriate timeline of treatment discontinuation, if necessary.

\section{Additional Information \\ Disclosures}

Human subjects: Consent was obtained or waived by all participants in this study. Conflicts of interest: In compliance with the ICMJE uniform disclosure form, all authors declare the following: Payment/services info: All authors have declared that no financial support was received from any organization for the submitted work. Financial relationships: All authors have declared that they have no financial relationships at present or within the previous three years with any organizations that might have an interest in the submitted work. Other relationships: All authors have declared that there are no other relationships or activities that could appear to have influenced the submitted work.

\section{Acknowledgements}

The completion of this undertaking could not have been possible without the participation and assistance of so many people whose names may not all be enumerated. Their contributions are sincerely appreciated and gratefully acknowledged. To all relatives, friends, and others who, in one way or another, shared their support, either morally, financially, and physically, thank you.

\section{References}

1. Keytruda: PD-1 receptor blockade. (2021). https://www.keytruda.com/hcp/mechansim-of-action/.

2. De Leo EK, Shah CP, Grajo JR, et al.: Extramedullary hematopoiesis in mismatch repair deficient colon cancer patient on adjuvant chemotherapy. Cureus. 2021, 13:e12899. 10.7759/cureus.12899

3. Yawalkar N, Pichler WJ: Mechanisms of cutaneous drug reactions [Article in German]. J Dtsch Dermatol Ges. 2004, 2:1013-1023. 10.1046/j.1439-0353.2004.04524.x

4. Keough GC, Richardson TT, Grabski WJ: Pravastatin-induced lichenoid drug eruption. Cutis. 1998, 61:98100.

5. Halevy S, Shai A: Lichenoid drug eruptions. J Am Acad Dermatol. 1993, 29:249-255. 10.1016/01909622(93)70176-t

6. Joseph RW, Cappel M, Goedjen B, et al.: Lichenoid dermatitis in three patients with metastatic melanoma treated with anti-PD-1 therapy. Cancer Immunol Res. 2015, 3:18-22. 10.1158/2326-6066.CIR-14-0134

7. Villadolid J, Amin A: Immune checkpoint inhibitors in clinical practice: update on management of immunerelated toxicities. Transl Lung Cancer Res. 2015, 4:560-575. 10.3978/j.issn.2218-6751.2015.06.06

8. Eggermont AM, Chiarion-Sileni V, Grob JJ : Adjuvant ipilimumab versus placebo after complete resection of 


\section{Cureus}

high-risk stage III melanoma (EORTC 18071): a randomised, double-blind, phase 3 trial. Lancet Oncol. 2015, 16:522-530. 10.1016/S1470-2045(15)70122-1

9. Hodi FS, O’Day SJ, McDermott DF, et al.: Improved survival with ipilimumab in patients with metastatic melanoma. N Engl J Med. 2010, 363:711-723. 10.1056/NEJMoa1003466

10. Cardis M.A, Jiang H, Strauss J, et al.: Diffuse lichen planus-like keratoses and clinical pseudo-progression associated with avelumab treatment for Merkel cell carcinoma. BMC Cancer. 2019, 539. 10.1186/s12885019-5759-1

11. McQuestion M: Evidence-based skin care management in radiation therapy: clinical update . Semin Oncol Nurs. 2011, 27:e1-e17. 10.1016/j.soncn.2011.02.009 